\title{
Sasso Pisano Geothermal Field Environment Harbours Diverse Ktedonobacteria Representatives and Illustrates Habitat-Specific Adaptations
}

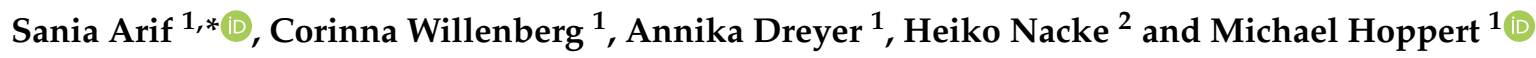 \\ 1 Department of General Microbiology, Institute of Microbiology and Genetics, University of Göttingen, \\ Grisebachstraße 8, 37077 Göttingen, Germany; corinna.willenberg@stud.uni-goettingen.de (C.W.); \\ annika.dreyer@stud.uni-goettingen.de (A.D.); mhopper@gwdg.de (M.H.) \\ 2 Department of Genomic and Applied Microbiology, Institute of Microbiology and Genetics, \\ University of Göttingen, Grisebachstraße 8, 37077 Göttingen, Germany; hnacke@gwdg.de \\ * Correspondence: sarif@gwdg.de; Tel.: +49-551-39-9657; Fax: +49-551-39-3808
}

check for updates

Citation: Arif, S.; Willenberg, C.; Dreyer, A.; Nacke, H.; Hoppert, M. Sasso Pisano Geothermal Field Environment Harbours Diverse Ktedonobacteria Representatives and Illustrates Habitat-Specific

Adaptations. Microorganisms 2021, 9, 1402. https://doi.org/10.3390/ microorganisms 9071402

Academic Editor: Ricardo Amils

Received: 9 June 2021

Accepted: 24 June 2021

Published: 29 June 2021

Publisher's Note: MDPI stays neutral with regard to jurisdictional claims in published maps and institutional affiliations.

Copyright: (c) 2021 by the authors. Licensee MDPI, Basel, Switzerland. This article is an open access article distributed under the terms and conditions of the Creative Commons Attribution (CC BY) license (https:/ / creativecommons.org/licenses/by/ $4.0 /)$.

\begin{abstract}
The hydrothermal steam environment of Sasso Pisano (Italy) was selected to investigate the associated microbial community and its metabolic potential. In this context, 16S and 18S rRNA gene partial sequences of thermophilic prokaryotes and eukaryotes inhabiting hot springs and fumaroles as well as mesophilic microbes colonising soil and water were analysed by high-throughput amplicon sequencing. The eukaryotic and prokaryotic communities from hot environments clearly differ from reference microbial communities of colder soil sites, though Ktedonobacteria showed high abundances in various hot spring samples and a few soil samples. This indicates that the hydrothermal steam environments of Sasso Pisano represent not only a vast reservoir of thermophilic but also mesophilic members of this Chloroflexi class. Metabolic functional profiling revealed that the hot spring microbiome exhibits a higher capability to utilise methane and aromatic compounds and is more diverse in its sulphur and nitrogen metabolism than the mesophilic soil microbial consortium. In addition, heavy metal resistance-conferring genes were significantly more abundant in the hot spring microbiome. The eukaryotic diversity at a fumarole indicated high abundances of primary producers (unicellular red algae: Cyanidiales), consumers (Arthropoda: Collembola sp.), and endoparasite Apicomplexa (Gregarina sp.), which helps to hypothesise a simplified food web at this hot and extremely nutrient-deprived acidic environment.
\end{abstract}

Keywords: Sasso Pisano; hot vents; fumarole; 16S rRNA gene; $18 \mathrm{~S}$ rRNA gene; microbial diversity

\section{Introduction}

The superheated steams and fumaroles near Sasso Pisano village (Castelnuovo Val di Cecina, Italy) are the central part of the Larderello geothermal field, located in the inner Northern Apennines (Southern Tuscany). The field exhibits extremely high subsurface temperatures $\left(300-350^{\circ} \mathrm{C}\right)$ at a pressure range of 4-7 $\mathrm{MPa}$ [1-3]. A vapour-dominated reservoir within a metamorphic basement at a depth of $3000 \mathrm{~m}$ is connected to a productive $1000 \mathrm{~m}$ deep carbonate-evaporite reservoir, which is almost sealed by overlying units. Many artificial steam wells were established for geothermal energy generation. The fumarolic fields of Sasso Pisano between Lagoni del Sasso and Monterotondo Marittimo represent a unique landscape of natural steam vents [3]. The overpressurised hot steam and springs release and/or redeposit $\mathrm{CH}_{4}, \mathrm{CO}_{2}$, sulphur compounds $\left(\mathrm{H}_{2} \mathrm{~S}, \mathrm{SO}_{4}{ }^{2-}\right)$, boric acid $\left(\mathrm{H}_{3} \mathrm{BO}_{3}\right)$, ammonia $\left(\mathrm{NH}_{4}{ }^{+}\right)$, and heavy metals at the surface [4,5], as water vapours flow $(50 \mathrm{t} / \mathrm{h})$ along fault zones / fractures through the sedimentary layers $[3,6]$. The condensation of sulphur dioxide-rich steam at the surface around fumaroles contributes to an acidic environment ( $\mathrm{pH} 0.5-5)$ [7], which also affects the surrounding area [8,9]. 
The Sasso Pisano geothermal field comprises very extreme environmental constraints including heated surface soil $\left(50\right.$ to $\left.100{ }^{\circ} \mathrm{C}\right)$, extremely low $\mathrm{pH}$ values, low content of bioavailable nitrogen, carbon and phosphorus compounds, and high content of heavy metals as well as trace elements, especially around fumaroles $[5,9,10]$. These stringent conditions would limit the growth and productivity of microbes and select only a few of the most adaptive extremophiles to survive in the extreme geothermal fields. The bacterial communities of this extreme environment are barely explored, though the extremophilic microorganisms are of great interest due to their potential biotechnological importance [11]. To learn more about the bacterial and eukaryotic communities in the presence of hot steams, environmental DNA from the geothermal fields in Sasso Pisano was isolated for 16S and 18S rRNA gene sequencing and subsequent diversity analysis. The study of the Sasso Pisano fumaroles' rock and soil samples revealed high predominance of extremophilic novel uncultured Ktedonobacteria and eukaryotic Cyanidiales. Furthermore, the adaptive strategies of Ktedonobacteria and Cyanidiales in relation to environmental constraints were inspected. We also analysed if the diversity of Ktedonobacteria at Sasso Pisano is similar to that determined in other environments.

\section{Materials and Methods}

\subsection{Sample Collection}

The samples were taken at Sasso Pisano (Pisa, Tuscany Region, Italy) in April $2019\left(43^{\circ} 09.632^{\prime}\right.$ North, $10^{\circ} 51.538^{\prime}$ East) and stored at $-20^{\circ}$ until further use. For prokaryotic community analysis, the sampling sites at the hot springs and nearby soil were sampled (see sample designations in Figure 1). The designation of the samples was SP (Sasso Pisano). The hot springs varied greatly in intensity and discharged whitish to blackish tarnish, accompanied by steam bubbling. Sample SP1 was taken from a greenish/brownish biofilm growing at the top of the hot spring A vent, constantly exposed to steam. Sample SP2 was taken from a yellowish/beige, possibly elemental sulphur deposit formed by vapours at the hot spring A. SP11 (greyish muddy watery discharge) and SP12 (yellowish greyish sediments) were directly sampled from the hot spring $B$ vent. The hot spring $C$ was sampled at different distances from the openings SP24, SP26 and SP7. The hot spring D was sampled at the vent (SP310) and when the discharge was meeting hot spring C sediments and organic material (plant leaves: SP38) (Figure 1D). A description of the features and details with respect to all samples are provided in Table S1. Soil samples (Sp513-515, Sp3, Sp517, Sp719, Sp819-821, Sp122) with various textures were frequently collected around the different hot springs. To study a thermophilic eukaryotic consortium, growing under extremely acidic $(\mathrm{pH} 2)$ and hot conditions $\left(44-55^{\circ} \mathrm{C}\right)$, a greenish biofilm (SP5) was collected from the vertical walls of a small gorge filled with hot steam seeping out of the ground (Figure $1 \mathrm{H}$ ). For comparison among the extreme sites, a water stream with a neutral $\mathrm{pH}$ and a lower temperature $\left(15^{\circ} \mathrm{C}\right)$ near the fumarole crossing the site was sampled and labelled as SP4 (control). 

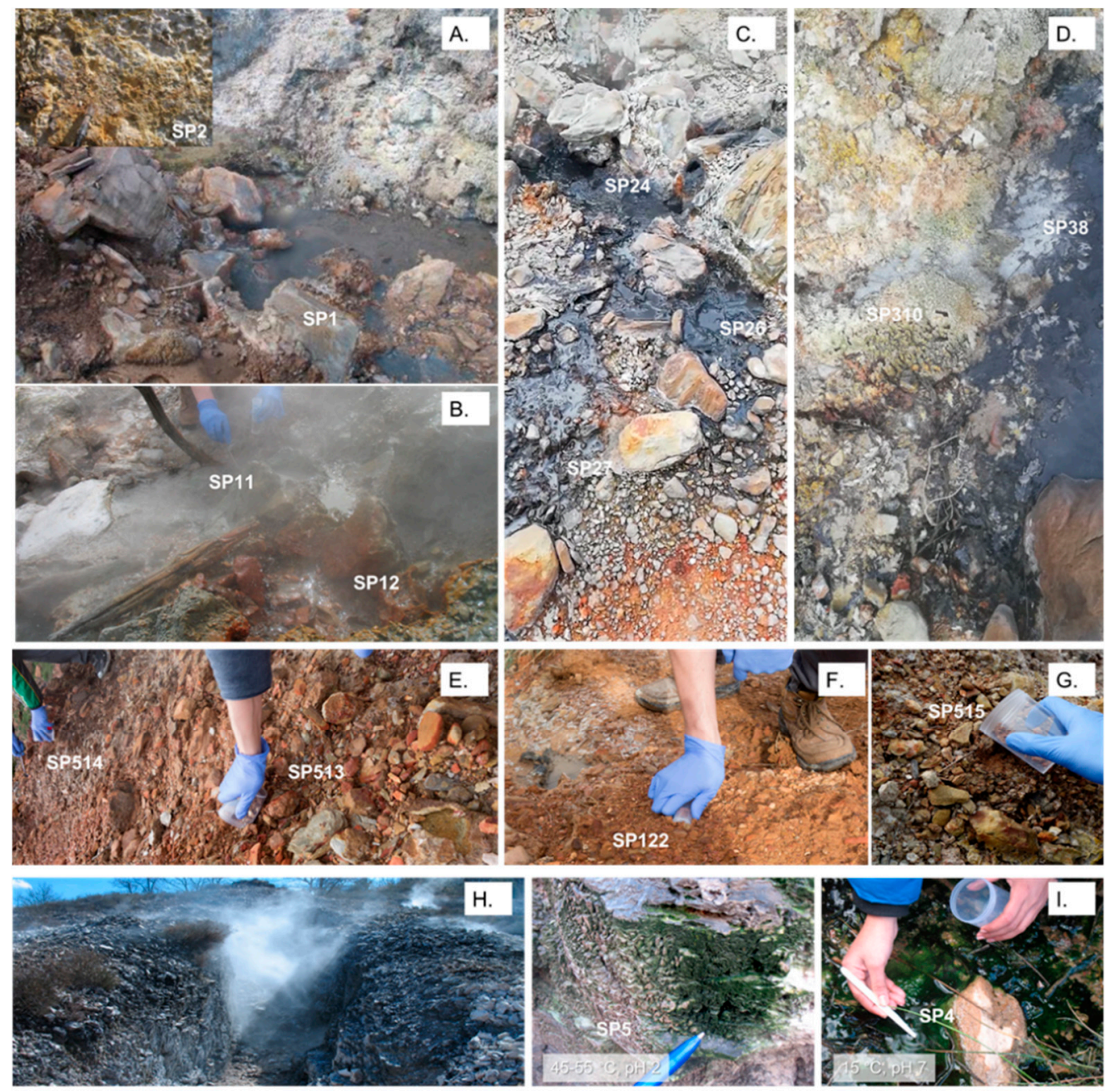

Figure 1. Sampling sites for 16S rRNA and 18S rRNA gene-based analysis. (A-D) are hot springs sites, while (E-G) are representatives of the soil sampling sites. Each sample is labelled "SP" (Sasso Pisano), followed by numbers. The picture inserted in (A) represents the yellowish-orange SP2 sampling site, formed by the surface deposition of the sulphur vapours probably from a solfatara-type vent. Hot spring sites (A,D) were either of clear or whitish liquid discharge. Hot springs $(\mathbf{B}, \mathbf{C})$ were splashing muddy greyish/blackish water. The fumarole rocky wall was continuously wrapped in hot steam (H). The greenish biofilm forms a tight layer on the crumbly rock of the fumarole (SP5) and was compared with a cold and pH-neutral water stream (SP4) (I).

\subsection{Environmental DNA Extraction, Amplicon Generation and Sequencing}

The Power soil DNA isolation kit (Qiagen, Hilden, Germany) was used to extract the whole genomic DNA of the stored samples. The samples were subjected to three extractions (triplicates) each, according to the manufacturer's protocol. The extracted gDNA was then concentrated with the Eppendorf Concentrator Plus (Eppendorf, Hamburg, Germany) at 45 or $60^{\circ} \mathrm{C}$ for $35 \mathrm{~min}$ to obtain the optimal gDNA concentration. Qualitative and quantitative determination of DNA was performed by gel electrophoresis and a Nanodrop instrument (NanoDrop 2000 c Spectrophotometer, ThermoFisher Scientific, Waltham, MA, USA). After quality assessment, partial $16 \mathrm{~S}$ and $18 \mathrm{~S}$ rRNA genes were amplified via polymerase chain reaction (PCR). For eukaryotes, the $18 \mathrm{~S}$ amplicon PCR forward primer $=5^{\prime}-\mathrm{TCG}$ TCG GCA GCG TCA GAT GTG TAT AAG AGA CAG CCA GCA SCY GCG GTA ATT CC- $3^{\prime}$ and the reverse primer $=5^{\prime}-$ GTC TCG TGG GCT CGG AGA TGT GTA TAA GAG ACA GAC TTT CGT TCT TGA TYR A-3' ${ }^{\prime}$ were used. For bacteria, the $16 \mathrm{~S}$ amplicon forward primer $5^{\prime}$-TCG TCG GCA GCG TCA GAT GTG TAT AAG AGA CAG CCT ACG GGN GGC WGC AG-3' and the reverse primer $=5^{\prime}$-GTC TCG TGG GCT CGG AGA TGT GTA TAA GAG ACA GGA 
CTA CHV GGG TAT CTA ATCC-3' were applied $[12,13]$. These primers were additionally linked to the overhang adapter sequences to make the amplicon suitable for Illumina MiSeq sequencing. Both partial $16 \mathrm{~S}$ and $18 \mathrm{~S}$ rRNA genes were amplified via a modified PCR Master mixture: Double-distilled nuclease-free water $(32 \mu \mathrm{L})$ was mixed with $10 \mu \mathrm{L}$ Phusion GC Buffer- $5 \times, 10 \mu \mathrm{M}$ forward and reverse primers $(1.0 \mu \mathrm{L}$ each $), 5 \%$ DMSO $(2.5 \mu \mathrm{L}), 50 \mathrm{mM}$ $\mathrm{MgCl}_{2}(0.15 \mu \mathrm{L}), 10 \mathrm{mM}$ dNTPs $(1.0 \mu \mathrm{L}), 25 \mathrm{ng}$ template DNA $(2.0 \mu \mathrm{L})$ and $0.5 \mu \mathrm{L}$ of $2 \mathrm{U} / \mu \mathrm{L}$ Phusion HF DNA polymerase (ThermoFisher Scientific) to obtain a final volume of $50 \mu \mathrm{L}$ reaction mixture. A thermal cycler (Biometra, Göttingen, Germany) was used to anneal the $18 \mathrm{~S}$ and $16 \mathrm{~S}$ rRNA gene-specific primers with the extracted gDNA at 56 and $60{ }^{\circ} \mathrm{C}$, respectively. PCR was performed as described $[13,14]$. The amplification products were qualitatively and quantitatively controlled on a $0.8 \%$ agarose gel with $1 \times$ TAE buffer (ThermoFisher Scientific) and a NanoDrop spectrophotometer (ThermoFisher Scientific). PCR products were further processed by using the GeneRead Size Selection Kit (Qiagen) to wash off primers and PCR reagents from the resulting amplicons. Indexing of OCR products was executed with the Nextera XT DNA library prep kit (Illumina, San Diego, CA, USA); subsequently, paired-end sequencing at the Göttingen Genomics Laboratory using the $2 \times 300 \mathrm{bp}$ Paired-End mode with an Illumina MiSeq sequencer (Illumina) was performed. The obtained Fastq sequences are accessible at the NCBI database under the Sasso Pisano microbiome project number PRJNA725822.

\subsection{Processing of $16 S$ rRNA Gene-Based Amplicon Sequencing Data}

The MetaAmp automated pipeline, an online resource for metagenomic analysis, was utilised to analyse $16 \mathrm{~S}$ rRNA gene-based amplicon sequencing data (http:/ / ebg.ucalgary. $\mathrm{ca} /$ metaamp/, accessed on 18 November 2020) [15]. Initially, the USEARCH software merged the uncompressed and demultiplexed sequence data and removed low-quality reads [16]. The subsequent step was the removal of non-matching and misaligned read pairs of small lengths (less than $350 \mathrm{bp}$ ). Moreover, the Mothur software package was utilised for the identification and trimming of forward and reverse primers [17]. Any reads that lacked forward and reverse primers or containing primer mismatches were discarded. In the next step, the reads were dereplicated using UPARSE software version 7.1 [18]. This software also discarded chimaeras and singletons. The resulting, high-quality reads were assembled into taxonomic units (OTUs) based on the 0.97 OTU clustering threshold ( $97 \%$ identity). The Mothur software package assigned taxonomic status to the OTUs with reference to the SILVA training dataset (http:/ / www.mothur.org/wiki/Taxonomy_outline, accessed on 18 November 2020). Lastly, the aforementioned software generated relative abundance data, rarefaction curves, as well as alpha and beta diversity indexes. The samples were rarefied into subsamples. The lack of similarity within different samples was computed through the Bray-Curtis index and principal coordinate analysis (PCoA) was performed. The Permutational Multivariate Analysis of Variance (PERMANOVA) test allowed for hypothesis testing.

\subsection{Analysis of $18 S$ rRNA Gene-Based Amplicon Sequencing Data}

With respect to analysis of $18 \mathrm{~S}$ rRNA gene-based data, paired-end reads were merged using PEAR (Paired-End reAd mergeR) [19] and resulting sequences of small lengths (less than $400 \mathrm{bp}$ ) were removed. Furthermore, the BBMap tool was used to remove all sequences that did not match the primer sequences at the $5^{\prime}$ and $3^{\prime}$ ends [20]. Subsequently, chimeric sequences were removed with the VSEARCH tool [21]. Data cleaned in this way were converted from the FASTQ file format to the FASTA format and mapped with QIIME (Quantitative Insights into Microbial Ecology) against the reference genes from the SILVA $13218 \mathrm{~S}$ database [22] with a $97 \%$ match [23]. 


\subsection{Transmission Electron Microscopy (TEM)}

For TEM sample preparation, the samples SP4 and SP5 were placed in a fixing solution $(0.2 \%, v / v$, glutardialdehyde, $0.3 \%, w / v$, formaldehyde). Subsequently, they were washed with a $50 \mathrm{mM} \mathrm{K}_{2} \mathrm{HPO}_{4}$ buffer solution and parts of the biofilm were embedded in agar (Agar-Agar, Kobe I, Carl Roth, Karlsruhe, Germany). Sliced small agar blocks of samples were dehydrated with ethanol in increasing concentration, followed by infiltration with synthetic resin (LR White Resin, London Resin Company, Berkshire, UK), and then, embedded in gelatine capsules, which were polymerised at $55^{\circ} \mathrm{C}$ for $24 \mathrm{~h}$.

To obtain ultra-thin sections, the polymerised samples were first pre-milled with a sample trimming device (TM 60, Reichert-Jung, Wetzlar, Germany), and sliced with the ultramicrotome (Ultracut E, Reichert-Jung) using a diamond knife (DuPont, Wilmington, DE, USA). The quality of the ultrathin sections was assessed based on their interference pattern and "fished" out of the water using coated grids (Plano, Wetzlar, Germany) as specimen support. For staining of ultrathin sections, uranyl acetate and replacement stain (UAR-EMS Uranyl Acetate Replacement Stain, Electron Microscopy Sciences, Hatfield, UK) were applied at different dilutions (undiluted, 1:3, 1:5, $v / v$ ). The grid with the section side facing downwards was placed on a drop of $20 \mu \mathrm{L}$ of the respective staining agent and was incubated for ten minutes. The ultrathin sections were examined by transmission electron microscopy (JEM-1011 Electron Microscopes, JEOL, Akishima, Tokyo, Japan) at a voltage of $80 \mathrm{kV}$ and electron micrographs were recorded.

\subsection{Prokaryotic Community Functional Profiling}

The MetaAmp-generated taxonomic profile data were utilised to generate a holistic overview of functional profiles of various samples. In this context, the Piphilin online server (http:/ / secondgenome.com/Piphillin, accessed on 5 October 2020) [24] was used. Piphilin allows for the direct search of representative OTU sequences against a database composed of $16 \mathrm{~S}$ rRNA gene sequences using USEARCH version 8.0.1623 [16]. In the next step, gene copy numbers of every inferred genome are summed to generate $\mathrm{KO}$ abundances (the KEGG reference database was used) [25]. The online available server MicrobiomeAnalyst [26] was utilised for the completion of statistical and meta-analysis of gene abundance data. The R package DESeq2 was used to compute a differential abundance analysis [27]. To summarise the findings, important features of hot sampling sites were considered in regard to soil samples that exhibited over a 1-fold difference.

\subsection{Phylogenetic Analysis}

The phylogenetic analysis was focused on 16S rRNA gene sequences, including those of different Ktedonobacteria type strains. MUSCLE, implemented in MEGA-X software version 7.0, was utilised to align these sequences and also Ktedonobacteria class-related OTU sequences [28]. Lastly, as part of the phylogenetic analyses, evolutionary distances were assessed. The Kimura 2-parameter model was used for this. The construction of phylogenetic trees was performed utilising the maximum likelihood method [29]. Bootstrap values were calculated based on 1000 replications.

\section{Results}

\subsection{Prokaryotic Community Composition}

A total of 788,808 high-quality $16 \mathrm{~S}$ rRNA gene sequences were available for analysis of prokaryotic communities. Per sample, the sequence amount ranged from 14,219 to 92,423 and a total of 1797 OTUs were identified (Figure S1). The sampling sites at the hydrothermal field were divided into two groups: hot springs and soils. The relative abundances at hot springs varied greatly per sample, emphasising each hot spring microbiome is unique (Figure 2). For instance, the prokaryotic profile of the samples SP24 and SP26, emerging from the same blackish muddy hot spring C, was dominated mainly by Aquificae ( $p=0.001083)$, and hot spring B SP11 and SP12 samples were dominated by Deinococcus/Thermus $\left(p=1.8017 \times 10^{-15}\right)$. Chloroflexi were abundant in the SP1 biofilm taken from hot spring A expelling clear water steam 
fumes, and samples SP11 and SP12 from slightly muddy water hot spring B. The phylumlevel profile also changed drastically when the hot spring streams (SP24, SP26 and SP310) were coming into contact with soil (SP27) or other organic matter (leaves, SP38) downstream, suggesting the integration of a mesophilic consortium (including Firmicutes or Proteobacteria). The overall composition of the hot spring samples comprised Chloroflexi (21\%), Proteobacteria $(19 \%)$, Firmicutes (17\%), Cyanobacteria (8\%), Aquificae (6\%), Acidobacteria (5\%), Deinococcus Thermus (4\%), Euryarchaeota (3\%), Thermotogae (6\%) and Actinobacteria (2\%). In contrast, in all soil samples, the most abundant taxa were Cyanobacteria (27\%), Proteobacteria (19\%), Acidobacteria (11\%), Actinobacteria (10\%), Chloroflexi (8\%), Euryarchaeota (7\%), Planctomycetes (6\%) and Thaumarchaeota (4\%) and the composition showed no drastic variations among most of the samples. An increased abundance of Chloroflexi was detected in soil samples that were collected nearby fumaroles or hot springs.

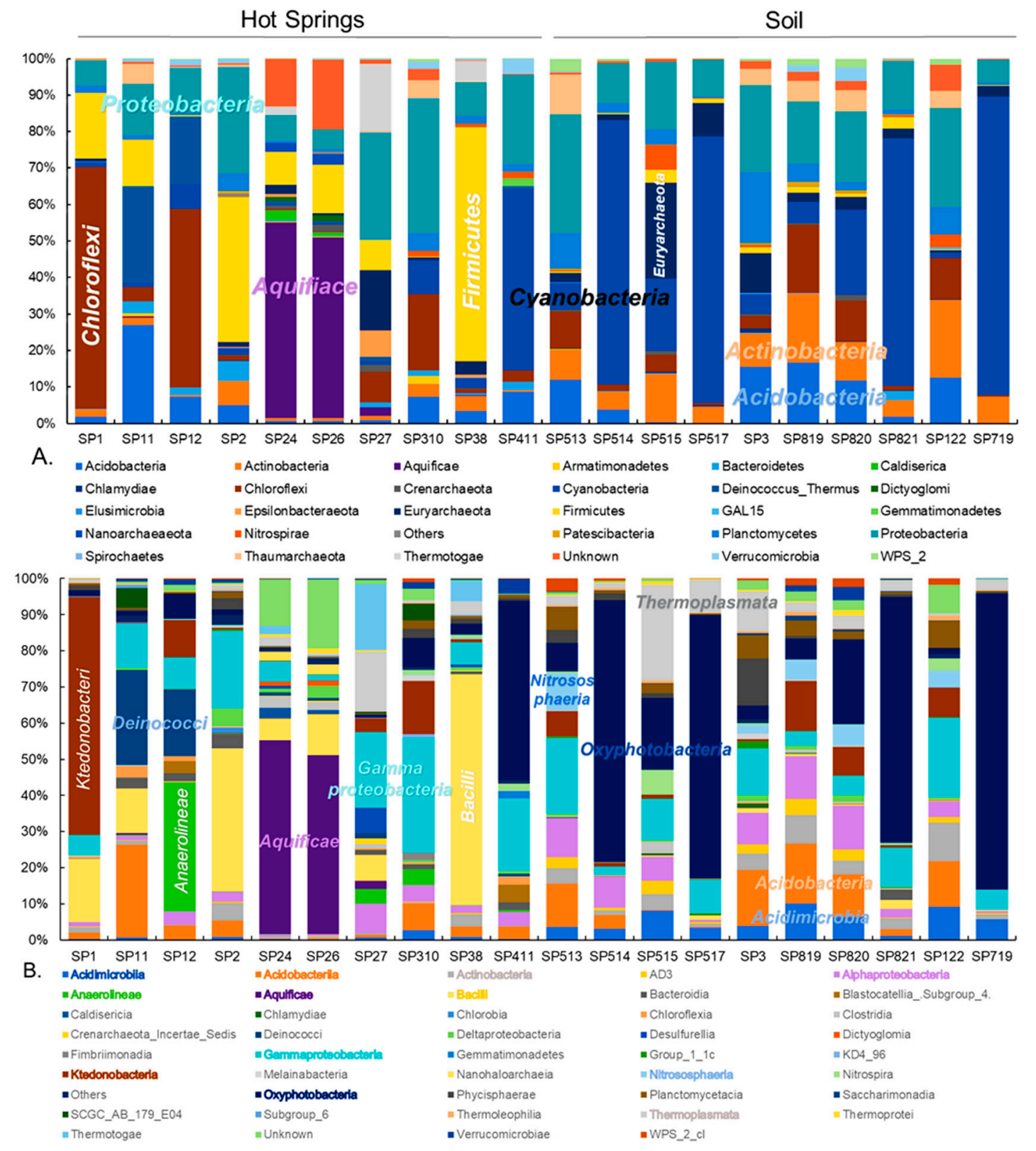

Figure 2. Relative abundance of prokaryotic taxa in Sasso Pisano hot spring and soil samples. Prokaryotic community composition at the phylum level (A) and class level (B) is shown for individual samples. Abundant taxa are highlighted within stacked bars or within taxa legends. 
At the class level, the differences between the hot spring samples were more prominent. For instance, the SP1 biofilm from hot spring A showed a high abundance of the branched hyphae and spore forming Ktedonobacteria (67\%), while the other hot spring sample SP12 was enriched with filamentous thermophilic Anaerolineae (36\%). Deinococci $(27 \%)$, Aquificae (54-50\%), Gammaproteobacteria (21-32\%), Acidobacteria (16\%), and Oxyphotobacteria (54\%) were the most abundant taxa in samples SP11, SP24, SP26, SP27 and SP310, respectively. Bacilli (Firmicutes) inhabited the surface of yellowish sulphur deposits (44\%, SP2) and a downstream site (65\%, SP38) where the whitish discharge mixed with leaves and blackish muddy discharge from hot spring $C$. In the group of soil samples, the abundant classes were, in different samples, Oxyphotobacteria, Thermoplasmata, Alphaproteobacteria and Gammaproteobacteria, Acidobacteria and Actinobacteria. The depicted pie charts show taxa in the hot spring and soil group in the order of ascending relative abundance (Figure 3). Overall, the hot spring group showed a higher relative abundance of thermophilic taxa.

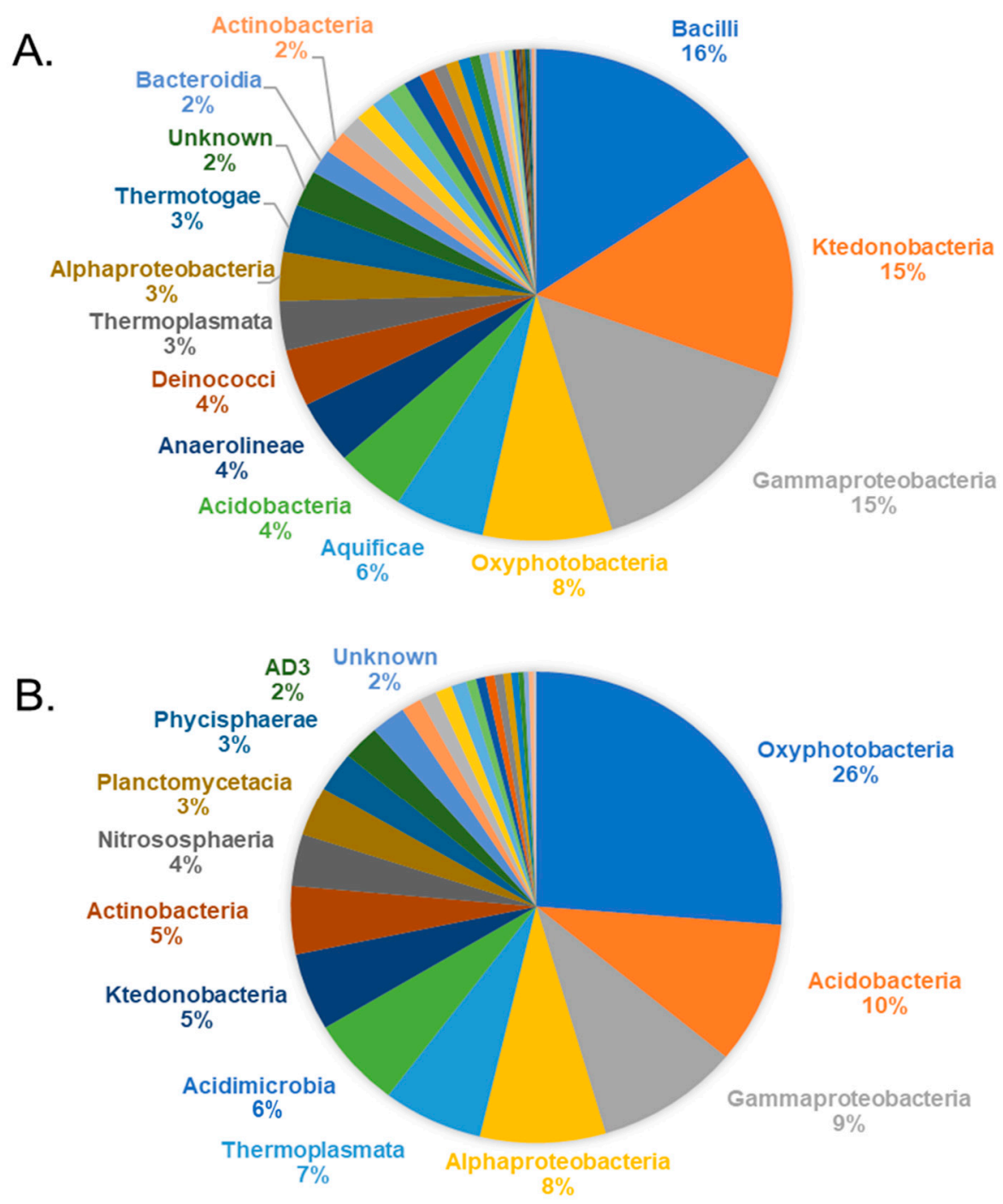

Figure 3. Overall bacterial community composition of the hot spring and soil microbiome. The actual abundances at the class level are plotted for the hot spring (A) and soil group (B). Taxa showing less than $2 \%$ relative abundance are not displayed. 


\subsection{Diversity of Ktedonobacteria}

The 30 most abundant OTU sequences potentially affiliated with Ktedonobacteria were aligned with $16 \mathrm{~S}$ rRNA gene sequences of isolated Ktedonobacteria strains and metagenomic sequences resulting from NCBI blast. In the phylogenetic tree, the OTUs are sandwiched between mesophilic (Dictyobacter) and thermophilic (Thermogemmatispora) groups of the Ktedonobacteria, indicating the presence of a large pool of uncultured mesophilic to thermophilic Ktedonobacteria strains. Most strains could be classified as members of Ktedonobacteraceae; however, OTUs related to JG30-KF-AS9 and B10-SB3A seem to be outliers and are more closely related to thermophilic Thermogemmatisporaceae (Figure 4). The detected OTUs indicate that the natural hydrothermal field of Sasso Pisano offers a rich reservoir of novel uncultured Ktedonobacteria, which should be targeted with respect to cultivation, genome sequencing, and further exploration. The highest abundance of Ktedonobacteraceae was detected in the SP1 biofilm, which indicates a natural enrichment of these bacteria at a hot spring vent (Figure S2). More diverse genus members belonging to Ktedonobacteria were identified in another hot spring sample (SP310; whitish discharge).

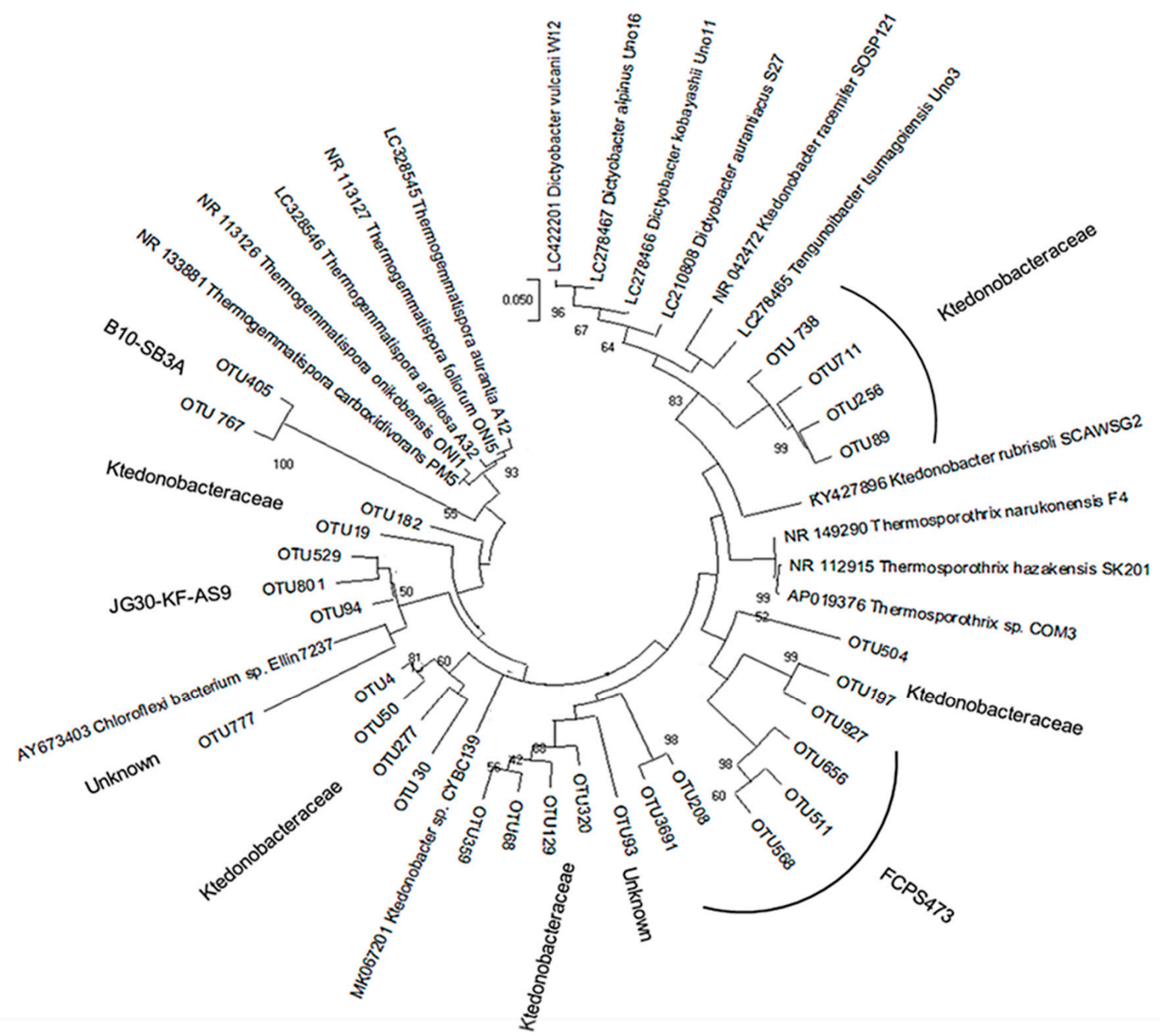

Figure 4. Maximum likelihood phylogenetic tree including Ktedonobacteria OTUs. The phylogenetic tree was constructed by aligning OTU sequences potentially affiliated with Ktedonobacteria with 16S rRNA gene sequences of known Ktedonobacteria species. 


\subsection{Alpha and Beta Diversity}

For alpha and beta diversity analysis, sequence datasets were rarefied to the lowest detected read size per sample. The Shannon index indicated that some of the hot spring samples comprise a lower diversity than soil group samples (Figure 5). The soil group potentially exhibited a higher alpha diversity in some cases, as the environmental conditions are comparably less harsh and facilitate a broad range of microorganisms to grow. In contrast, due to the acidic $\mathrm{pH}$, higher temperature, and constant washout at the hot spring spots, only acidic thermophilic microbes are supposed to grow. However, the $t$-test result does not show a significant difference between the two groups, indicating that the soil microbial communities may provide an active microbial influx to the hot springs and vice versa (Figure 5A).
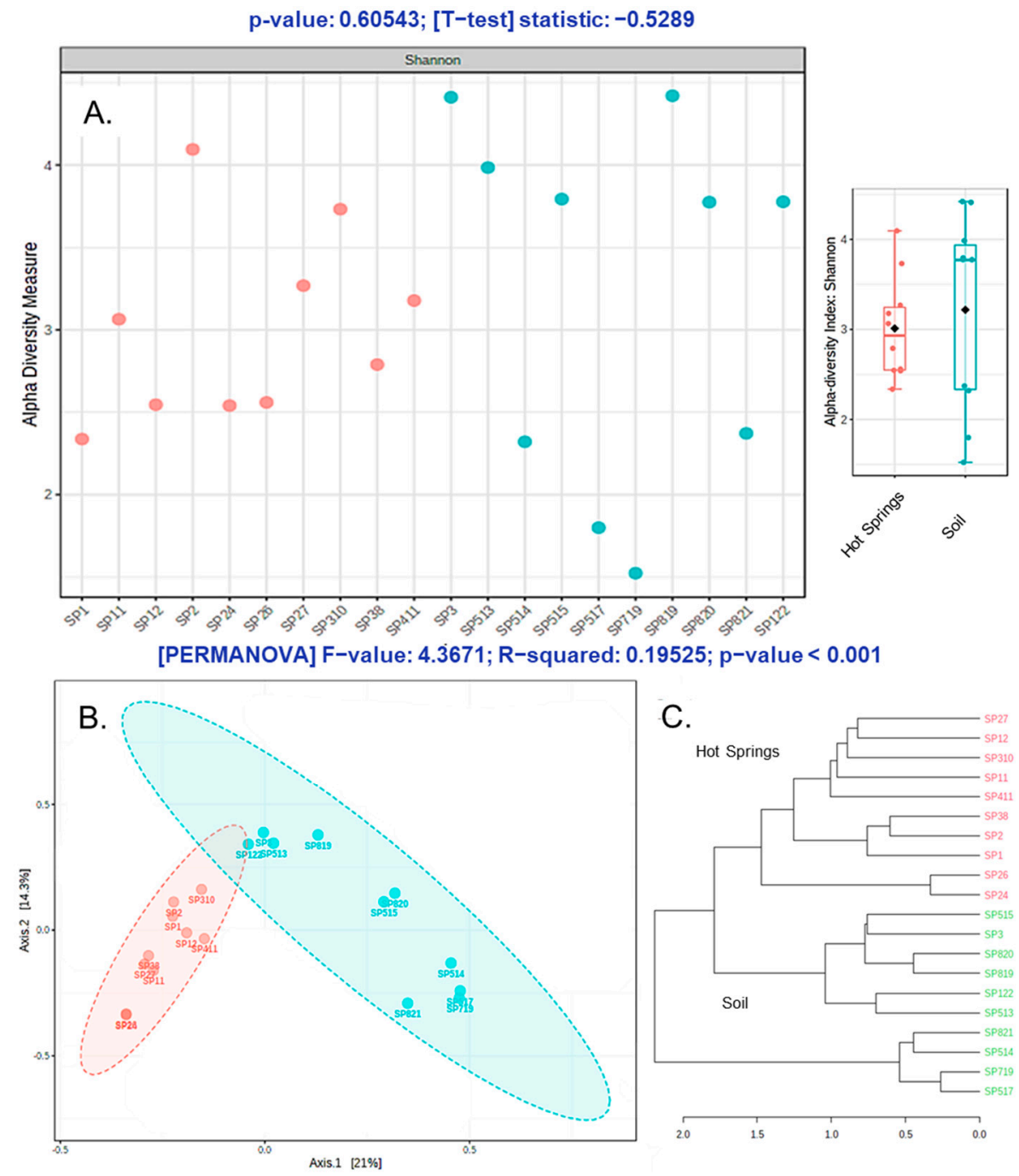

Figure 5. Comparative alpha and beta diversity analysis of the hot springs versus soil samples. The Shannon index (A) showed that both hot springs and soil samples were rich in terms of microbial alpha diversity, though the particular microbial composition for both habitats was distinct, based on the PCoA (B) and Bray-Curtis tree (C) analysis.

To visualize and explore the complex metagenomic data, the multidimensional scaling method-Principal Coordinates Analysis (PCoA) - was applied to observe the similarities at the OTU level. The PCoA of Sasso Pisano samples showed that the microbial communities of hot springs and soil samples were clustering separately, with a considerable variance among the two groups (Figure 5B). According to the Permutational Multivariate Analysis of Variance (PERMANOVA), the beta diversity among both groups also suggested the 
inhabitant microbial communities are distinct, indicating the stringent effect of hot spring constraints in selection and enrichment of a unique microbial consortium as compared to the soil microflora. The tree diagram, calculated based on a Bray-Curtis index, also indicated that both groups are clearly distinct (Figure 5C).

\subsection{Functional Profile}

The abundance of functional genes summarised by $\mathrm{KO}$ identifiers (also called K numbers) was estimated from the OTU data. Based upon this estimation, the predicted function of genes was used to evaluate a metabolic profile of the microbial communities in different sites. The differential abundance analysis of KO numbers indicated that the hot spring microbiota was metabolically rich in terms of heavy metal resistance, methane, sulphur and nitrogen metabolism as well as aromatic compounds' degradation pathways as compared to the soil microbial consortium. Numerous transporters involved in the extrusion of $\mathrm{Mn}$, $\mathrm{Zn}, \mathrm{Cu}, \mathrm{W}, \mathrm{Co}, \mathrm{Ni}$ and Mo were present in the hot spring microbial consortium (Table 1). The functional profile also showed that the different $\mathrm{KO}$ numbers associated with methane metabolism are differentially present in hot spring and soil samples (Figure 6). This indicates that the hot spring microbiome utilises different enzymes as compared to the soil microbiome to metabolise environmental methane. The soil microbiome shows preferences for aerobic methane metabolism such as methane oxidation to formaldehyde and formate dehydrogenation to $\mathrm{CO}_{2}$, while the hot spring microbiome seems to favour anaerobic methane metabolism through reverse-methanogenesis by means of methyl-coenzyme $\mathrm{M}$ reductase (Mcr-AOM) [30,31] (Figure S3). Moreover, with respect to carbon fixation, mainly formaldehyde seems to be fixed via the ribulose-monophosphate pathway (RuMP) [32]. More KO numbers in the hot spring microbial communities were also observed to be differentially abundant, involved in sulphur and nitrogen metabolism (data not shown). In the aromatic compound degradation pathways, genes and enzymes involved in the aminobenzoate, benzoate, chlorocyclohexane, chlorobenzene, chloroalkane, chloroalkene, dioxin, fluorobenzoate, nitrotoluene, naphthalene, styrene, xylene and toluene degradation were also differentially abundant in the hot spring samples (Figure S4).

Table 1. Differentially abundant transporters required for heavy metal resistance in hot springs microbiome.

\begin{tabular}{|c|c|}
\hline \multicolumn{2}{|c|}{ Transport System } \\
\hline $\begin{array}{c}\text { Manganese } \\
\text { K19973 mntA; manganese ATP-binding protein } \\
\text { K19975 mntC; manganese substrate-binding protein } \\
\text { K19976 mntB; manganese permease protein } \\
\text { K11601 mntC; manganese substrate-binding protein } \\
\text { K11603 mntA; manganese ATP-binding protein } \\
\text { K11602 mntB; manganese permease protein }\end{array}$ & $\begin{array}{l}\text { K11707 troA; manganese/zinc/iron substrate-binding protein } \\
\text { K11708 troC; manganese/zinc/iron permease protein } \\
\text { K11709 troD; manganese/zinc/iron permease protein } \\
\text { K11710 troB; manganese/zinc/iron ATP- binding protein }\end{array}$ \\
\hline $\begin{array}{c}\text { Iron } \\
\text { K11604 sitA; manganese/iron substrate-binding protein } \\
\text { K11605 sitC; manganese/iron permease protein } \\
\text { K11606 sitD; manganese/iron permease protein } \\
\text { K11607 sitB; manganese/iron ATP-binding protein } \\
\text { K02010 afuC; iron(III) ATP-binding protein } \\
\text { K02011 afuB; iron(III) permease protein } \\
\text { K02012 afuA; iron(III) substrate-binding protein }\end{array}$ & $\begin{array}{c}\text { Copper } \\
\text { K19340 nosF; Cu-processing system ATP-binding protein } \\
\text { K19341 nosY; Cu-processing system permease protein } \\
\text { Tungstate } \\
\text { K05772 tupA; tungstate substrate-binding protein } \\
\text { K05773 tupB; tungstate permease protein }\end{array}$ \\
\hline $\begin{array}{l}\text { Molybdate } \\
\text { K02017 modC; molybdate ATP-binding protein } \\
\text { K02018 modB; molybdate permease protein } \\
\text { K02020 modA; molybdate substrate-binding protein }\end{array}$ & $\begin{array}{c}\text { Nickel } \\
\text { K15584 nikA; nickel substrate-binding protein } \\
\text { K15585 nikB; nickel permease protein } \\
\text { K15586 nikC; nickel permease protein } \\
\text { K15587 nikD; nickel ATP-binding protein }\end{array}$ \\
\hline $\begin{array}{l}\text { Sulphate/Thiosulphate } \\
\text { K02045 cysA; sulfate/thiosulfate ATP-binding protein } \\
\text { K02046 cysU; sulfate/thiosulfate permease protein } \\
\text { K02047 cysW; sulfate/thiosulfate permease protein } \\
\text { K02048 cysP; sulfate/thiosulfate substrate-binding protein }\end{array}$ & $\begin{array}{l}\text { Cobalt } \\
\text { K02006 cbiO; cobalt/nickel ATP-binding protein } \\
\text { K02007 cbiM; cobalt/nickel permease protein } \\
\text { K02008 cbiQ; cobalt/nickel permease protein } \\
\text { K02009 cbiN; cobalt/nickel transport protein }\end{array}$ \\
\hline
\end{tabular}




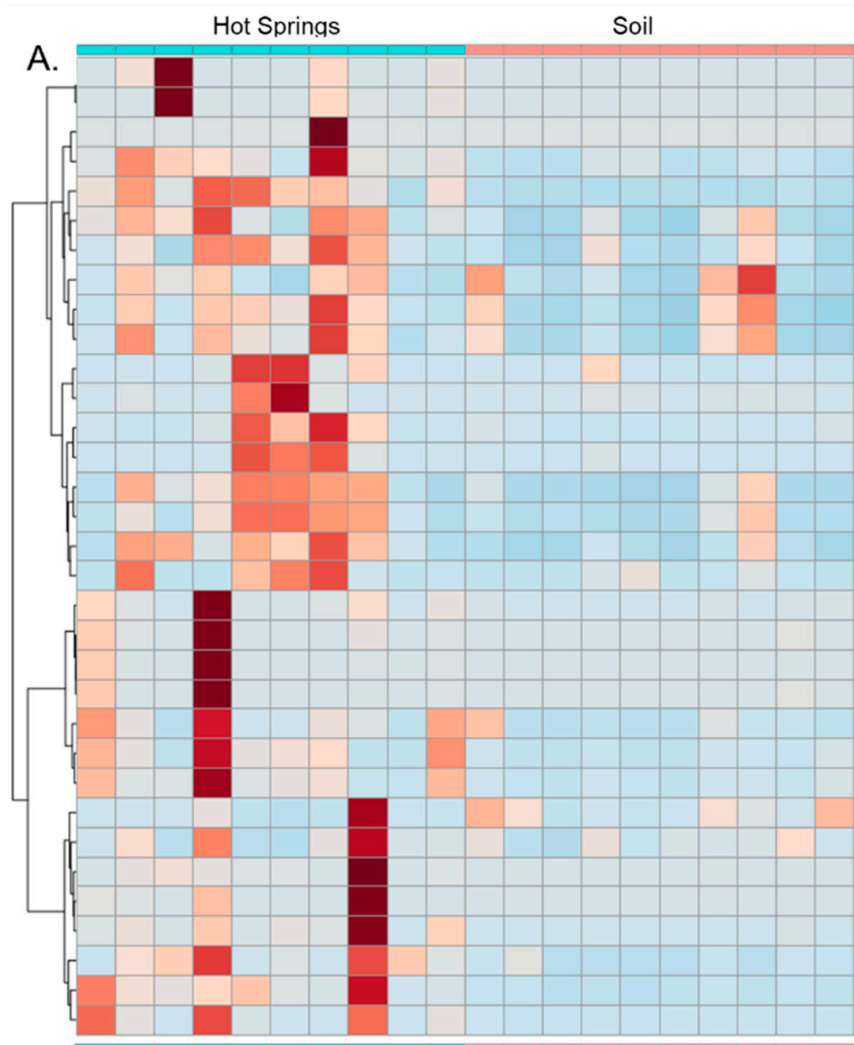

K117807,8-didemethyl-8-hydroxy-5-deazariboflavin synthase [EC:4.3.1.32] K117815-amino-6. (D-ribitylamino) uracil-L-tyrosine 4-hydroxyphenyl transferase K001892-0xoisovalerate/pyruvate ferredoxin oxidoreductase gamma subunit[EC:1.2.7.7] K059792-phosphosulfolactate phosphatase [EC:3.1.3.71] KO0850 6-phosphofructokinase 1 [EC:2.7.1.11]

K01624 fructose-bisphosphate aldolase, class II [EC:4.1.2.13]

K018342,3-bisphosphoglycerate-dependent phosphoglycerate mutase [EC:5.4.2.11] K01595 phosphoenolpyruvate carboxylase [EC:4.1.1.31] K01689 enolase [EC:4.2.1.11]

K01895 acetyl.COA synthetase [EC:6.2.1.1]

KO0170 pyruvate ferredoxin oxidoreductase beta subunit[EC:1.2.7.1] KO3390 heterodisulfide reductase subunit C2 [EC:1.8.7.31.8.98.41.8.98.51.8.98.6] KO0018 glycerate dehydrogenase [EC:1.1.1.29]

KO0171 pyruvate ferredoxin oxidoreductase delta subunit [EC:1.2.7.1] K00124 formate dehydrogenase iron-sulfur subunit K00127 formate dehydrogenase subunit gamma

K01007 pyruvate, water dikinase [EC:2.7.9.2]

K01622 fructose 1,6-6iphosphate adolase [EC:4.1.2.133.1.3.11]

K00863 triose/dihydroxyacetone kinase / FAD-AMP lyase (cyclizing) [EC:2.7.1.282.7.1.29]

K07812 trimethylamine-N-oxide reductase (cytochrome C) [EC:1.7.2.3]

K03532 trimethylamine-N-oxide reductase (cytochrome c), cytochrome c-type subunit TorC K03533 TorA specific chaperone

K00148 glutathione-jndependent formaldehyde dehydrogenase [EC:1.2.1.46]

K080933-hexulose-6-phosphate synthase [EC:4.1.2.43]

K080946-phospho-3-hexuloisomerase [EC:5.3.1.27]

K01499 methenyltetrahydromethanopterin cyclohydrolase [EC:3.5.4.27]

K01623 fructose-bisphosphate aldolase, class I [EC:4.1.2.13]

K13039 sulfopyruvate decarboxylase subunit beta [EC:4.1.1.79]

KO2203 phosphoserine / homoserine phosphotransferase [EC:3.1.3.32.7.1.39]

K14083 trimethylamine---corrinoid protein Co-methyltransferase [EC:2.1.1.250]

K06034 sulfopyruvate decarboxylase subunit alpha [EC:4.1.1.79]

K00584 tetrahydromethanopterin S-methyltransferase subunit $H$ [EC:2.1.1.86]

K08685 quinohemoprotein amine dehydrogenase [EC:1.4.9.1]

B.

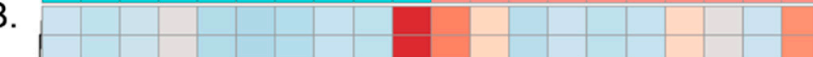

K14028 mdh1; methanol dehydrogenase (cytochromec) subunit 1 [EC:1.1.2.7]

K14029 mdh2; methanol dehydrogenase (cytochromec) subunit2 [EC:1.1.2.]

K08692 mtKB; malate-CoA ligase subunit alpha [EC:6.2.1.9]

K14067 mtkA; malate-COA ligase subunit beta [EC:6.2.1.9]

$\mathrm{K} 00300 \mathrm{mtd}$; methylenetetrahydrofolate/methylenetetrahydromethanopterin dehydrogenase (NADP+)

K16255 mxaG; cytochrome c-L

K01623ALDO; fructose-bisphosphatealdolase, class | [EC:4.1.2.13]

K10713 fae; 5,6,7,8-tetrahydromethanopterin hydro-yase [EC:4.2.1.147]

KO0201 fwdB; formylmethanofuran dehydrogenase subunit B [EC:1.2.7.12]

KO0202 fwdC; formylmethanofuran dehydrogenase sub unit $C[E C: 1.2 .7 .12]$

KO0200 fwdA; formylmethanofuran dehydrogenase subunit A [EC: 1.2.7.12]

K09733 mfnB; (5-formylfuran-3-y)||methyl phosphate synthase [EC:4.2.2.153]

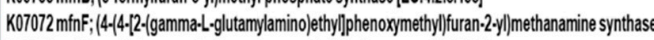

K10714 mtdB; methylene-tetrahydromethanopterin dehydrogenase [EC:1.5.1]

K07144 mfnE; 5. -aminomethyl)-3.furanmethanol phosphate kinase [EC:2.7.4.31]

KO0672 ftr; formylmethanofuran-tetrahydromethanopterin $\mathrm{N}$-formyltransferase [EC:23.1.101]

K01499 mch; methenyltetrahydromethanopterin cyclohydrolase [EC:3.5.4.27]

K03396 gra; S.(hydroxymethyl)glutathione synthase [EC:4.4.1.22]

K10946 pmoC-amoC; methanelammonia monooxygenase subunitC

K10944 pmoA-amoA; methanelammonia monooxygenase subunitA [EC:1.14.18.31.14.99.39]

K10945 pmoB-amoB; methanelammonia monooxygenase subunit B

K01079 serB; phosphoserine phosphatase [EC:3.1.3.3]

K00122 FOH; formate dehydrogenase [EC:1.17.1.9]

K00577 mtrA; tetrahydromethanopterin S-methyltransferase subunitA [EC:21.1.86]

K18933 mfnA; tyrosine decarboxylase/ aspartate 1-decarboxylase [EC:4.1.1.254.1.1.11]

K22482 hdrC1; heterodisulfide reductase subunitC1 [EC:1.8.7.3]

K12234 coff; coenzyme F420-0:L-glutamate ligase / coenzyme F420-1:gamma-L-glutamate ligase

K11212 cofD; LPPG:FO 2-phospho-L-Lactatet transferase [EC:27.8.28]

K14941 cofC; 2-phospho-L-lactate/ phosphoenolpyruvate guanylyltransferase [EC:2.7.7.68 2.7.7.105]

$\mathrm{K} 22087 \mathrm{mgdD}$; methylglutamate dehydrogenase subunitD [EC:1.5.99.5]

K22085 mgdB; methylglutamate dehydrogenase subunit B [EC:1.5.99.5]

K22084 mgdA; methylglutamate dehydrogenase subunit A [EC:1.5.99.5

$\mathrm{K} 22086 \mathrm{mgdC}$; methylglutamate dehydrogenase subunitC [EC:1.5.99.5]

K22083 mgsC; methylamine---glutamate N-methyltransferase subunit C [EC:2.1.1.21]

K22081 mgsA; methylamine--.glutamate N-methyltransferase subunitA [EC:21.1.21]

K22082mgsB: methylamine---glutamate N-methyltransferase subunit B [EC:2.1.1.21]

K17067 mdo; formaldehyde dismutase / methanol dehydrogenase [EC:1.2.98.11.1.99.37]

Ko0320 mer; 5,10 -methylenetetrahydromethanopterin reductase [EC:1.5.98.2]

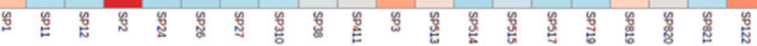

K18277 tmm; trimethylamine monooxygenase [EC:1.14.13.148]

Figure 6. Differentially abundant genes involved in methane metabolism. The hot spring microbiome (A) was found to be abundant in the genes involved in the anaerobic methane degradation as compared to the aerobic methane degradation in the soil microbiome $(\mathbf{B})$. 


\subsection{Eukaryotic Diversity at a Fumarole}

According to rarefaction curves, 300-450 OTUs were identified in the neutral $\mathrm{pH}$ and mesophilic stream conditions; the extreme fumarole site comprised less than 50 OTUs (Figure S5). A total of 57 OTUs affiliated with known taxonomic groups were identified in stream sample (SP4) data, while the fumarole rock (SP5) data comprised a total of 21 groups, which shows a considerably higher alpha diversity in the water stream biome than in the fumarole biome. It can be concluded that the extreme conditions of the fumarole limit microbial alpha diversity, while microbial communities could easily populate the neutral $\mathrm{pH}$ water stream.

Organisms known to typically colonise freshwater habitats, including amoebae $(11.2 \%)$ which can be divided into the groups Dactylopodida (1.1\%), Filamoeba $(7 \%)$ and Ischnamoeba sp. $(2.3 \%)$, were detected. The Incerta Sedis represents a kind of placeholder for unspecified species in the genome database. The paraphyletic group of green algae is represented by Chlorophyta $(0.75 \%)$ and Charophyta $(4 \%)$. Among the multicellular organisms, Chaetonotida $(10 \%)$, belonging to the phylum Gastrotricha ("hairybacks"), was detected. Furthermore, Adineta vaga, belonging to the rotifers, was found with $2.1 \%$ and the lower fungi were represented with a total of $5.7 \%$ relative abundance. The group of SAR (Stramenopiles, Alveolata, Rhizaria) showed $19.7 \%$ and LEMD267 is listed in SILVA as a nonspecific taxonomic group that refers to lobose amoebae $(22.5 \%)$. However, this cannot be further determined with the available data. Excluding the unspecified eukaryotes (19\%), 62.02\% of the identified species OTUs (Amoeba 22.5\%, Archaeplastida 0.82\%, Metazoa 14.3\%, SAR 19.7\%, low mushrooms $4.7 \%$ ) can be assigned to a biofilm in a freshwater area with a neutral $\mathrm{pH}$ and a lower temperature around $15^{\circ} \mathrm{C}$. The other major abundant taxa were Intramacronucleata (Ciliates) (16\%) and Chaetonotida (10\%). The OTU assigned to Rhodophyceae with $0.07 \%$ and Echinamoeba thermarum with $4.2 \%$ can be potentially explained by the association of the stream channel to the thermo-acidic sampling site, since both OTUs possess a thermophilic character.

The apparently qualitative identical species composition of the sample duplicates (SP4.1 and SP4.2) differs especially in terms of the fraction of nematode- and mite-related OTUs (Figure 7). The unspecified Cyanidiaceae and the species Galdieria sulphuraria belonging to the family Cyanidiales of phototrophic eukaryotic algae represent the most abundant taxa of the fumarole biofilm (65.3\%). Unspecified Collembola (springtails) and Folsomia candida contributed to Collembola sp. (16.8\%). Collembola sp. (Arthropoda: Hexapoda) represent more highly developed organisms, as they are among the typical bottom dwellers and have been described for other extreme sites, in particularly hot and particularly cold areas [33]. Gregarina caledia (Apicomplexa) contributed 14\% of the biofilm consortium (Figure 7).

\subsection{Structural and Morphological Description of a Eukaryotic Biofilm}

The electron micrographs of a fumarole biofilm (SP5) preparation allowed inspection of individual cells, which are separated from each other by a cell wall of approx. $0.2 \mu \mathrm{m}$ thickness. Within these cells, organelles, chloroplasts, in particular, are visible (Figure 8). Inside chloroplasts, membrane stacks of the thylakoids are prominent features. Densely packed cells (Figure 8A) are separated from each other by an extracellular matrix formed between the organisms. Remineralisation processes have caused dark precipitates to settle as small particles in the extracellular matrix. Larger particles are separated from the cells by the matrix (Figure 8A). The marked particles imitate the shape of the matrix.

Based on the number of spore cells within the mother cell, potentially either the genus Galdieria or Cyanidium could be identified. More than four spores in the cell represent a distinct structural feature of Galdieria (Figure 8B), while a sporangium with tetraspores could be classified as Cyanidium (Figure 8C). Based on the species description in Ciniglia et al. (2004) [34], the organism (Figure 8D,E) can be morphologically determined even more precisely as Galdieria sulphuraria under the electron microscope. The cell has a thick cell wall and contains at least seven visible chloroplast shapes in the cytosol, surrounding a central vacuole, visible as a membrane-bounded empty lumen. Ultrathin sections also reveal 
the coexistence of the bacterial colony outside the extracellular matrix of the Cyanidiaceae biofilm (Figure 8F).

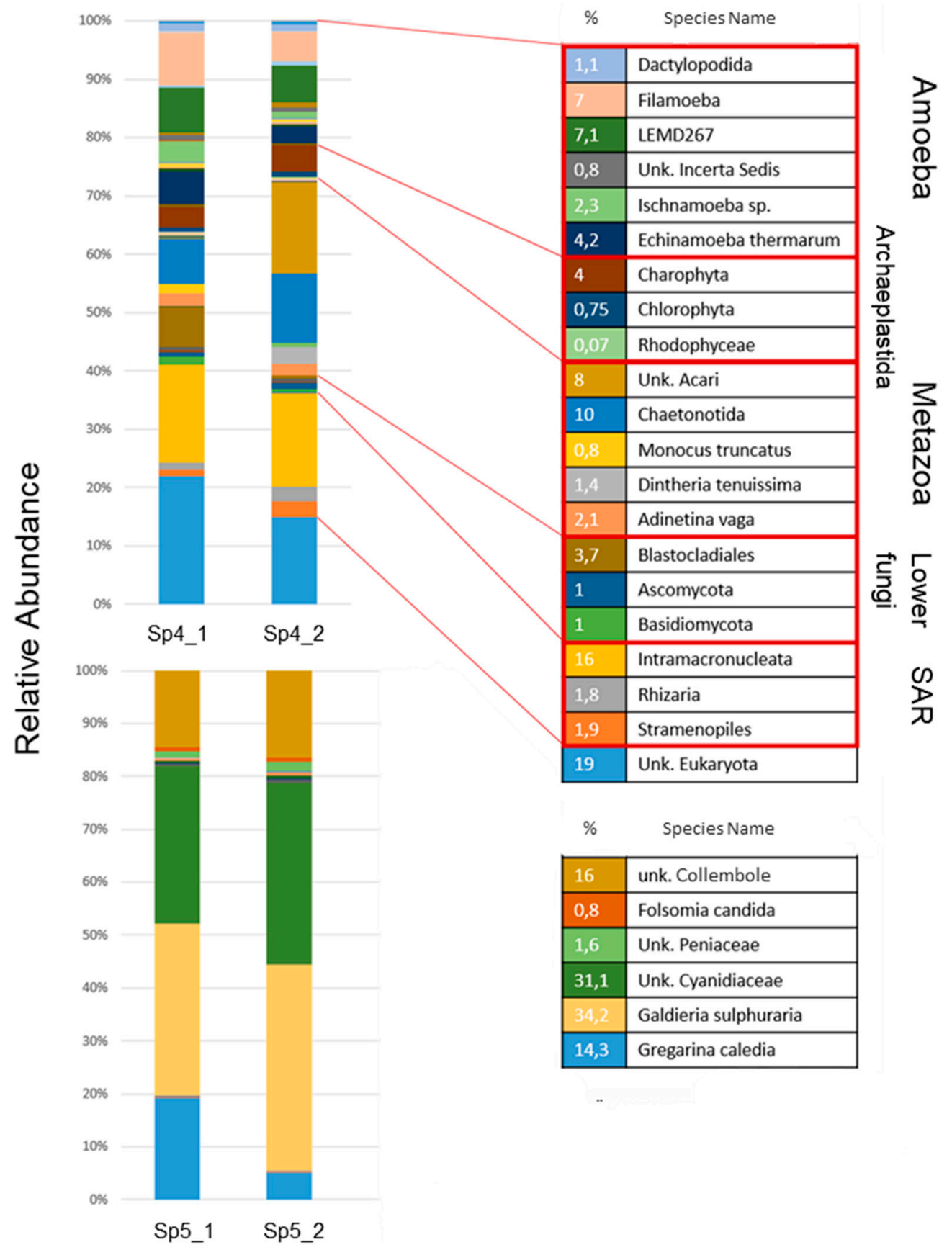

Figure 7. Eukaryotic community composition of a neutral $\mathrm{pH}$ (water stream biofilm SP4) and extreme (fumarole biofilm SP5) biome. Taxonomic groups are listed which show at least $0.80 \%$ of the total OTU abundance (except for the Rhodophyceae $(0.07 \%)$ from the stream water sample to show the contrast to the fumarole and Chlorophyta to distinguish them from Charophyta). In the stream water sample, 21 species groups are listed which represent $98.02 \%$ abundance of the total OTUs, and six groups are shown in the fumarole samples, which represent $97.90 \%$ of the total OTUs (Unk, unknown). 


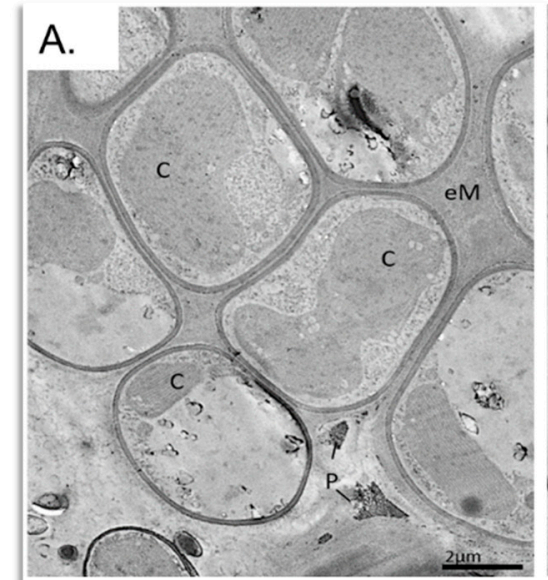

D.

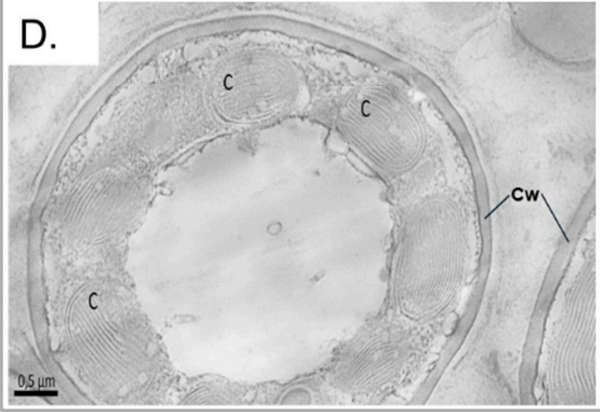

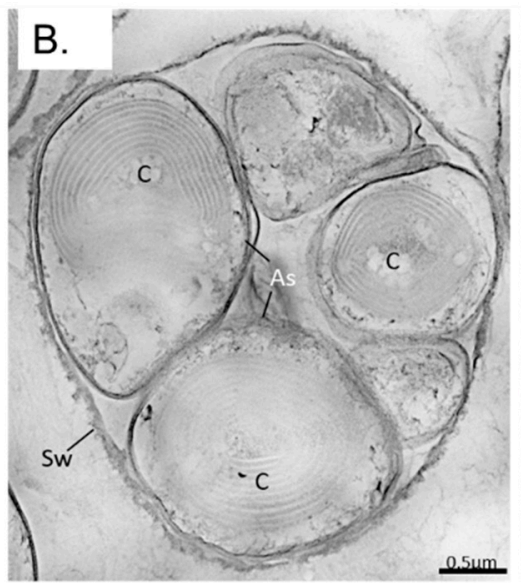

E.

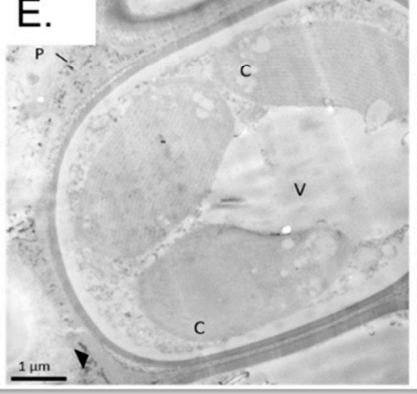

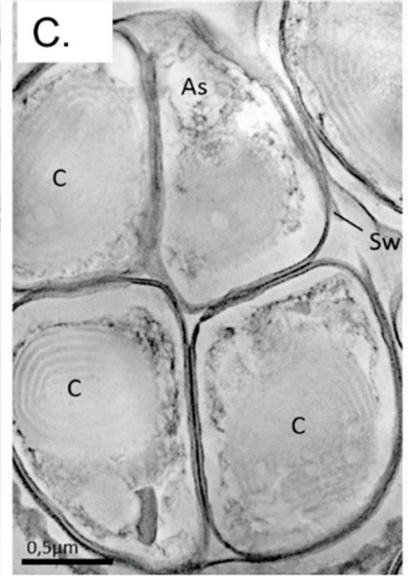

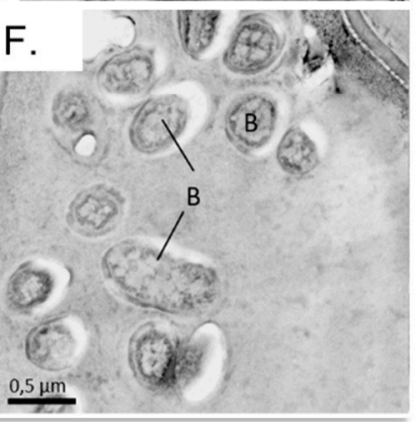

Figure 8. Electron microscopic analysis of a section through a sample taken from the fumarole biofilm SP5. An overview of a Cyanidiales cell aggregate (A). Sporangia (mother cells; B,C) containing a varying number of autospores. In three of the five autospores (B), the membrane stacks of the thylakoids in the chloroplasts are clearly visible. Within the mother cell (C), four autospores (tetraspores) already are about the same size and the sporangium wall is disintegrating. The cells in (D,E) carry multiple chloroplasts around a central vacuole. Both cells could be assigned to the genus Galdieria. Prokaryote morphotypes of about $0.5 \mu \mathrm{m}$ in size attached to the Cyanidiales aggregate (F). V, vacuole; eM, extracellular matrix; Cw, cell wall; Sw, sporangium wall; B, bacteria; As, autospores; P, precipitates.

\section{Discussion}

Ktedonobacteria, a deeply branched bacterial class comprising of mesophilic and thermophilic representatives, is characterised by its ubiquitous prevalence in terrestrial environments, complex life cycle, and in some cases, large genome size [35]. The class is divided into two orders, Ktedonobacterales and Thermogemmatisporales. The latter order includes Thermosporotrichaceae as well as Thermogemmatisporaceae strains, whereas Ktedonobacterales comprise Dictyobacteraceae, Ktedonobacteraceae and Ktedonosporobacteraceae strains. Within these two orders, 20 proposed mesophilic and thermophilic species have been assigned to the genera Dictyobacter, Tengunoibacter, Ktedonobacter, Ktedonosporobacter, Thermosporothrix and Thermogemmatispora [36-39]. Ktedonobacteria isolates and related environmental DNA are derived from non-extreme sources [38,39] and extreme environments, such as an acid vapour-formed spring [40], naturally occurring $\mathrm{CO}_{2}$ gas vents [41], a lava cave in a volcanic trench [42], volcanic fumaroles [43], steaming geothermal soil [44], and a mineral precipitating cave environment [45]. This suggests that Ktedonobacteria members appear to prevail in oligotrophic and extreme environments, implying these strains may have evolved versatile metabolic pathways to cope with extreme conditions. For instance, the potential to oxidise carbon monoxide (CO) has been reported for Ktedonobacteria members [46].

In this study, we gained insights into the abundance and diversity of Ktedonobacteria in the geologically diverse and environmentally extreme hydrothermal field environments of Sasso Pisano. Microbial diversity varied greatly with the type of sampling site, which included hot springs, a fumarole, and nearby soil as well as water samples. A high abundance and diversity of Ktedonobacteria at the hot springs and in some soil samples indicated that 
the Sasso Pisano hydrothermal region offers a natural reservoir of Ktedonobacteria members, which became naturally enriched under stringent environmental constraints including heated surface soil, extremely low $\mathrm{pH}$ values, low nutritional content of nitrogen, carbon and phosphorus and high content of heavy metals as well as trace elements, especially around fumaroles $[5,9,10]$. The functional profile indicated that the hot spring microbiome includes a significantly higher abundance of organisms harbouring genes involved in methane, sulphur, nitrogen and aromatic compounds' metabolism, since the acidic hot springs and fumaroles emit a mixture of various gases such as ammonia, methane, carbon dioxide, hydrogen, hydrogen sulphide, hydrocarbons and aromatic compounds [2,5,47]. Moreover, transporters conferring resistance against toxic transition metals were also detected in the hot spring microbiome, which implies that Ktedonobacteria members inhabiting this site could have evolved high metabolic plasticity to cope with acidification, nutrient depletion, and heavy metal resistance. In our previous study of biofilm samples from Marsberg copper mine, Germany, the capability to degrade toxic aromatic compounds as well as resistance against transition metals was detected in a metagenome-assembled genome affiliated with Ktedonobacteria [48]. The bacterial biofilm on steam vents from Sapichu volcanoes was also mainly colonised by related similar taxa of Chloroflexi (Ktedonobacteria), Acidobacteria, and Cyanobacteria. The metabolic potential analysis predicted similar cellular metabolic pathways related to methanogenesis, sulphur respiration, nitrogen fixation, and heavy metal transport except for photosynthesis by Cyanobacteria [43]. The observed higher abundance of Cyanobacteria in low-temperature soil samples (but not in hot springs samples) was consistent with colonisation patterns of Cyanobacteria in lower temperature vents, as reported by Wall et al. [49], while the higher temperature vents were abundant in Cloroflexi $[49,50]$.

Two important metabolic pathways in oligotrophic geothermal sites are attributed to trace gas $\left(\mathrm{CO}\right.$ and $\left.\mathrm{H}_{2}\right)$ utilisation by the type I carbon monoxide dehydrogenase (cox genes) and the [NiFe]-hydrogenase (hyp genes) as an energy source for cell growth and persistence under nutrient-limiting conditions [51]. Mounting genomic evidence suggests that three phyla, Chloroflexi, Actinobacteria and Acidobacteria, use $\mathrm{CO}$ and $\mathrm{H}_{2}$ as substrates [52]. A variety of thermophilic bacteria belonging to the classes Actinobacteria, Deinococci, Ktedonobacteria, Thermomicrobia and Clostridia contain cox operons and may be capable of aerobic $\mathrm{CO}$ oxidation. Hot springs from different regions host different cox encoding communities [53]. The Ktedonobacteria genome also includes the reductive TCA cycle along with multiple copies of cox operons, conferring CO oxidation potential to this taxa, allowing it to predominate the microbial community [46] under the influence of CO-rich gas vents, hydrothermal springs, and soil environments $[41,54,55]$. Other high abundant taxa have evolved diverse strategies, for instance, most Anaerolineaceae species metabolise various organic carbon sources under anaerobic conditions through fermentative metabolism [56]. Representatives of the thermophilic Aquificae grow in hot springs via oxidation of dissolved ferrous iron or iron-containing minerals and conduct nitrogen fixation even at $70{ }^{\circ} \mathrm{C}[57,58]$. Deinococci are resistant to several stresses due to their highly efficient DNA damage repair ability and detoxification of several toxic compounds through hydrolytic activity $[59,60]$. Some Oxyphotobacteria (photosynthetic Cyanobacteria) are metabolically diverse primary producers and pioneer the colonisation where light is available including ecosystems with low light, low levels of $\mathrm{O}_{2}$ and/or sulfidic conditions, because of their ability to perform, besides oxygenic photosynthesis, anoxygenic photosynthesis [61,62].

The eukaryotic alpha diversity at the fumarole was significantly lower than in the river water sample, whereas the alpha diversity of prokaryotes did not vary significantly in the hot springs and soil samples. This indicates that thermophilic eukaryotic organisms are less diverse than thermophilic prokaryotes. Cyanidiales dominated the biofilm with an OTU of $65.3 \%$, as they represent the only phototrophic organisms growing under these conditions and have been extensively studied since the 1980s [63]. The Cyanidiales form a monophyletic group within the red algae, which inhabits the acidic hot springs at different sites. Cyanidiales were identified under the electron microscope based on the chloroplasts 
and reproduction pattern, as the genera Cyanidium and Galdieria. Cyanidium sp. propagate with tetraspores, whereas Galdieria is larger and forms more autospores and vacuoles than Cyanidium [64]. Yoon et al. (2006) described the structural composition of endolithic and interlithic populations of Cyanidiales at Pisciarelli in the Phlegraean fields near Naples, in Sasso Pisano (distinct from the site sampled in this study) and Monte Rotondo [65]. Overall, Galdieria dominated the fumaroles of Sasso Pisano, which was expected due to the mixotrophy, the resulting adaptation to fluctuating environmental factors, and availability of carbon to be exploited. Cyanidium caldarium was also identified.

Current descriptions of the organisms around geothermally active areas around the world usually only illuminate the photoautotrophic community of the order Cyanidiales without considering the heterotrophic eukaryotes. The investigation of the entire diversity is interesting as the extreme conditions can serve as models for living conditions in the early evolutionary stages of soil ecosystems and can provide further information on the development of biocoenoses in the history of the earth. On the other hand, they represent a reduced biocoenosis that can serve as a model for more complex ecosystems. Collembola sp. (Hexapoda) represent the largest and most complex species at this site. Collembola are among the typical bottom dwellers and have been described for other extreme sites, in particularly hot and cold areas [34]. They are known as consumers of bacteria, algae, and fungi [66-68], actively feeding and thriving on these biomass sources. Gregarina sp. are known to endoparasitise Hexapoda among invertebrates in numerous colonies [69]. Based on the detected taxa, a simple food web could be assumed in which Cyanidium and Galdieria, belonging to unicellular red algae Cyanidiales, represent the primary producers and build biomass which may be consumed by Collembola sp. as consumers, and Gregarina sp. endoparasitise Collembola sp. Symbiotic bacteria may facilitate the biofilm growth of Cyanidiales. Collembola sp. could alter the microbial communities, either directly (through selectively feeding) or indirectly (through the dissemination of microbial propagules). Ultimately, a more detailed description of the species for this site would be required to confirm the described food web as a next step. This can then serve as a model to describe the influences and interactions of the organisms involved on each other and relate these to more complex ecosystems.

\section{Conclusions}

The composition and morphology of microbiota around hot fluid springs, steam vent and soil samples from the geothermally active hydrothermal fields around the Sasso Pisano site (Pisa, Tuscany region) are presented. The prokaryotic community analysis indicated that Ktedonobacteria at the Sasso Pisano site ranged from thermophilic to mesophilic, and phylogenetically as well as metabolically diverse representatives were detected. Metabolic functional profiling of the thermophilic prokaryotic microbiota predicted a higher capability to utilise carbon (methane and aromatic compounds), sulphur and nitrogen compounds, and heavy metal resistance-conferring genes were also significantly more abundant in the hot spring microbiome as compared to the mesophilic soil microbial consortium. The diversity of eukaryotic microorganisms within biofilms at extreme conditions, with very high temperature $\left(55^{\circ} \mathrm{C}\right)$ and a very low $\mathrm{pH}$ value of 2 , was evaluated. The detection of red algae Cyanidiales, Arthropoda (Collembola sp.) and Apicomplexa (Gregarina sp.) proposed a simplified food web at the thermophilic extremely nutrient-deprived acidic environment of a fumarole.

Supplementary Materials: The following are available online at https: / www.mdpi.com/article / 10.3390/microorganisms9071402/s1, Table S1: Complete details of the sampling sites, Figure S1: Rarefaction curve, Figure S2: Number of sampled reads of the Ktedonobacteria members. Figure S3: Differentially abundant genes involved in the methane metabolism. Figure S4: Relative abundance of differentially abundant genes involved in the aromatic compounds metabolism. Figure S5. Eukaryotic rarefaction curve. 
Author Contributions: Conceptualization, M.H. and H.N.; methodology, A.D., C.W. and S.A.; validation, S.A., H.N. and M.H.; formal analysis, S.A.; investigation, S.A., C.W. and A.D.; resources, M.H.; Writing-Original draft preparation, S.A. and C.W.; Writing-Review and editing, H.N., A.D. and M.H.; visualization, S.A.; supervision, M.H.; funding acquisition, S.A. All authors have read and agreed to the published version of the manuscript.

Funding: This research was funded by a DAAD Doctoral research grant granted to S.A. and the APC was funded by the Open Access Publication Fund of the University of Göttingen.

Data Availability Statement: The amplicon sequencing data presented here is accessible at the NCBI database under the Sasso Pisano microbiome project number PRJNA725822.

Acknowledgments: The authors would like to acknowledge the support and assistance in sequencing by the Göttingen Genomics laboratory staff members.

Conflicts of Interest: The authors declare no conflict of interest.

\section{References}

1. Barelli, A.; Bertini, G.; Buonasorte, G.; Fiordelisi, A.; Fiordelisi, C. Recent deep exploration results at the margins of the LarderelloTravale geothermal system. In Proceedings of the World Geothermal Congress 2000, Kyushu, Japan, 28 May-10 June 2000.

2. Bartoli, G.; Bottega, S.; Forino, L.M.C.; Ciccarelli, D.; Spano, C. Plant adaptation to extreme environments: The example of Cistus salviifolius of an active geo-thermal alteration field. C. R. Biol. 2014, 337, 101-110. [CrossRef]

3. Bertini, G.; Casini, M.; Gianelli, G.; Pandeli, E. Geological structure of a long-living geothermal system, Larderello, Italy. Terra Nova 2006, 18, 163-169. [CrossRef]

4. Narasimhan, T.; Goyal, K. Subsidence due to geothermal fluid withdrawal. Subsid. Geotherm. Fluid Withdraw. 1982, 6, 35-36. [CrossRef]

5. Bussotti, F.; Tognelli, R.; Montagni, G.; Borghini, F.; Bruschi, P.; Tani, C. Response of Quercus pubescens leaves exposed to geothermal pollutant input in southern Tuscany (Italy). Environ. Pollut. 2003, 121, 349-361. [CrossRef]

6. Pippucci, A.; Lorenzi, R.; Spanò, C.; Sorce, C. Stress-induced changes to the flora in a geothermal field in central Italy. Acta Physiol. Plant 2015, 37, 1-10. [CrossRef]

7. Brogi, A. The structure of the Monte Amiata volcano-geothermal area (Northern Apennines, Italy): Neogene-Quaternary compression versus extension. Intl. J. Earth Sci. 2007, 97, 677-703. [CrossRef]

8. Zelenski, M.; Taran, Y.; Galle, B. High emission rate of sulfuric acid from Bezymianny volcano, Kamchatka. Geophys. Res. Lett. 2015, 42, 7005-7013. [CrossRef]

9. D'Amore, F.; Panichi, C. Evaluation of deep temperatures of hydrothermal systems by a new gas geothermometer. Geochim. Cosmochim. Acta 1980, 44, 549-556. [CrossRef]

10. Selvi, F. Geothermal Biotopes in Central Western Italy from a Botanical Viewpoint. Ecosystem Responses to CO2: The MAPLE Project Results; Official Publications of the European Communities; European Communities: Luxemburg, 1999.

11. Elmarsdottir, A.; Ingimarsdottir, M.; Hansen, I.; Olafsson, J.S.; Olafsson, E. Vegetation and invertebrates in three geothermal areas in Iceland. In Proceedings of the International Geothermal Conference, Reykjavik, Iceland, 14-17 September 2003; Citeseer: Reykjavik, Iceland, 2003.

12. Rothschild, L.J.; Mancinelli, R.L. Life in extreme environments. Nat. Cell Biol. 2001, 409, 1092-1101. [CrossRef]

13. Klindworth, A.; Pruesse, E.; Schweer, T.; Peplies, J.; Quast, C.; Horn, M.; Glöckner, F.O. Evaluation of general 16S ribosomal RNA gene PCR primers for classical and next-generation sequencing-based diversity studies. Nucleic Acids Res. 2013, 41, e1. [CrossRef] [PubMed]

14. Stoeck, T.; Bass, D.; Nebel, M.; Christen, R.; Jones, M.D.M.; Breiner, H.-W.; Richards, T.A. Multiple marker parallel tag environmental DNA sequencing reveals a highly complex eukaryotic community in marine anoxic water. Mol. Ecol. 2010, 19, 21-31. [CrossRef] [PubMed]

15. Amin, N.; Schneider, D.; Hoppert, M. Bioleaching potential of bacterial communities in historic mine waste areas. Environ. Earth Sci. 2018, 77, 542. [CrossRef]

16. Dong, X.; Kleiner, M.; Sharp, C.E.; Thorson, E.; Li, C.; Liu, D.; Strous, M. Fast and simple analysis of MiSeq amplicon sequencing data with MetaAmp. Front. Microbiol. 2017, 8, 1461. [CrossRef]

17. Edgar, R.C. Search and clustering orders of magnitude faster than BLAST. Bioinformatics 2010, 26, 2460-2461. [CrossRef]

18. Schloss, P.D.; Westcott, S.L.; Ryabin, T.; Hall, J.R.; Hartmann, M.; Hollister, E.B.; Lesniewski, R.A.; Oakley, B.B.; Parks, D.H.; Robinson, C.J.; et al. Introducing mothur: Open-source, platform-independent, community-supported software for describing and comparing microbial communities. Appl. Environ. Microbiol. 2009, 75, 7537-7541. [CrossRef] [PubMed]

19. Edgar, R.C. UPARSE: Highly accurate OTU sequences from microbial amplicon reads. Nat. Methods 2013, 10, 996-998. [CrossRef]

20. Zhang, J.; Kobert, K.; Flouri, T.; Stamatakis, A. PEAR: A fast and accurate Illumina Paired-End reAd mergeR. Bioinformation 2014, 30, 614-620. [CrossRef]

21. Bushnell, B. BBMap: A fast, accurate, splice-aware aligner. In Proceedings of the 9th Annual Genomics of Energy \& Environment Meeting, Walnut Creek, CA, USA, 20 March 2014; Lawrence Berkeley National Lab: Walnut Creek, CA, USA, 2014. 
22. Rognes, T.; Flouri, T.; Nicholas, B.; Quince, C.; Mahe, F. VSEARCH: A versatile open source tool for metagenomics. PeerJ 2016, 4, e2584. [CrossRef] [PubMed]

23. Quast, C.; Pruesse, E.; Yilmas, P.; Gerken, J.; Schweer, T.; Yarza, P.; Peplies, J.; Glockner, F.O. The Silva ribosomal RNA gene database project: Improved data processing and web-based tools. Nucleic Acids Res. 2013, 41, D590-D596. [CrossRef] [PubMed]

24. Comeau, A.M.; Douglas, G.M.; Langille, M.G.I. Mikrobiome Helper: A custom and streamlined workflow for microbiome research. Meth. Protoc. 2017, 2, 11.

25. Iwai, S.; Weinmaier, T.; Schmidt, B.L.; Albertson, D.G.; Poloso, N.J.; Dabbagh, K.; DeSantis, T.Z. Piphillin: Improved prediction of metagenomic content by direct inference from human microbiomes. PLoS ONE 2016, 11, e0166104. [CrossRef]

26. Kanehisa, M.; Sato, Y.; Kawashima, M.; Furumichi, M.; Tanabe, M. KEGG as a reference resource for gene and protein annotation. Nucleic Acids Res. 2016, 44, D457-D462. [CrossRef]

27. Dhariwal, A.; Chong, J.; Habib, S.; King, I.L.; Agellon, L.B.; Xia, J. MicrobiomeAnalyst: A web-based tool for comprehensive statistical, visual and meta-analysis of microbiome data. Nucleic Acids Res. 2017, 45, W180-W188. [CrossRef]

28. Love, M.I.; Huber, W.; Anders, S. Moderated estimation of fold change and dispersion for RNA-seq data with DESeq2. Genome Biol. 2014, 15, 550. [CrossRef]

29. Stecher, G.; Tamura, K.; Kumar, S. Molecular Evolutionary Genetics Analysis (MEGA) for macOS. Mol. Biol. Evol. 2020, 37, 1237-1239. [CrossRef] [PubMed]

30. Saitou, N.; Nei, M. The neighbor-joining method: A new method for reconstructing phylogenetic trees. Mol. Biol. Evol. 1987, 4, 406-425. [CrossRef] [PubMed]

31. Nazem-Bokaee, H.; Gopalakrishnan, S.; Ferry, J.G.; Wood, T.K.; Maranas, C.D. Assessing methanotrophy and carbon fixation for biofuel production by Methanosarcina acetivorans. Microb. Cell Factor. 2016, 15, 1-13. [CrossRef]

32. Bennett, R.K.; Steinberg, L.M.; Chen, W.; Papoutsakis, E.T. Engineering the bioconversion of methane and methanol to fuels and chemicals in native and synthetic methylotrophs. Curr. Opin. Biotechnol. 2018, 50, 81-93. [CrossRef] [PubMed]

33. Hwang, I.Y.; Nguyen, A.D.; Nguyen, T.T.; Nguyen, L.T.; Lee, O.K.; Lee, E.Y. Biological conversion of methane to chemicals and fuels: Technical challenges and issues. Appl. Microbiol. Biotechnol. 2018, 102, 3071-3080. [CrossRef]

34. Sømme, L. Invertebrates in Hot and Cold Arid Environments; Springer: Berlin/Heidelberg, Germany, $1995 ;$ p. 275.

35. Ciniglia, C.; Yoon, H.S.; Pollio, A.; Pinto, G.; Bhattacharya, D. Hidden biodiversity of the extremophilic Cyanidiales red algae. Mol. Ecol. 2004, 13, 1827-1838. [CrossRef]

36. Zheng, Y.; Saitou, A.; Wang, C.-M.; Toyoda, A.; Minakuchi, Y.; Sekiguchi, Y.; Ueda, K.; Takano, H.; Sakai, Y.; Abe, K.; et al. Genome Features and Secondary Metabolites Biosynthetic Potential of the Class Ktedonobacteria. Front. Microbiol. 2019, 10, 893. [CrossRef] [PubMed]

37. Yabe, S.; Aiba, Y.; Sakai, Y.; Hazaka, M.; Yokota, A. Thermosporothrix hazakensis gen. nov., sp. nov., isolated from compost, description of Thermospo-rotrichaceae fam. nov. within the class Ktedonobacteria Cavaletti et al. 2007 and emended description of the class Ktedonobacteria. Int. J. Syst. Evol. Microbiol. 2010, 60, 1794-1801. [CrossRef]

38. Yabe, S.; Aiba, Y.; Sakai, Y.; Hazaka, M.; Yokota, A. Thermogemmatispora onikobensis gen. nov., sp. nov. and Thermogemmatispora foliorum sp. nov., isolated from fallen leaves on geothermal soils, and description of Thermogemmatisporaceae fam. nov. and Thermogemmatisporales ord. nov. within the class Ktedonobacteria. Int. J. Syst. Evol. Microbiol. 2011, 61, 903-910.

39. Cavaletti, L.; Monciardini, P.; Bamonte, R.; Schumann, P.; Rohde, M.; Sosio, M.; Donadio, S. New Lineage of filamentous, spore-forming, Gram-positive bacteria from soil. Appl. Environ. Microbiol. 2006, 72, 4360-4369. [CrossRef] [PubMed]

40. Yabe, S.; Sakai, Y.; Abe, K.; Yokota, A.; Také, A.; Matsumoto, A.; Sugiharto, A.; Susilowati, D.; Hamada, M.; Nara, K.; et al. Dictyobacter aurantiacus gen. nov., sp. nov., a member of the family Ktedonobacteraceae, isolated from soil, and emended description of the genus Thermosporothrix. Int. J. Syst. Evol. Microbiol. 2017, 67, 2615-2621. [CrossRef] [PubMed]

41. Jiang, Z.; Li, P.; Jiang, D.; Dai, X.; Zhang, R.; Wang, Y.; Wang, Y. Microbial community structure and arsenic biogeochemistry in an acid vapor-formed spring in Tengchong Geothermal Area, China. PLoS ONE 2016, 11, e0146331. [CrossRef]

42. De Miera, L.E.S.; Arroyo, P.; de Luis Calabuig, E.; Falagan, J.; Ansola, G. High-throughput sequencing of 16S RNA genes of soil bacterial communities from a naturally occurring $\mathrm{CO}_{2}$ gas vent. Int. J. Greenh. Gas. Control. 2014, 29, 176-184. [CrossRef]

43. Northup, D.; Melim, L.; Spilde, M.; Hathaway, J.; Garcia, M.; Moya, M.; Stone, F.; Boston, P.; Dapkevicius, M.; Riquelme, C. Lava cave microbial communities within mats and secondary mineral deposits: Implications for life detection on other planets. Astrobiology 2011, 11, 601-618. [CrossRef]

44. Brito, E.M.S.; Romero-Núñez, V.M.; Caretta, C.A.; Bertin, P.; Valerdi-Negreros, J.C.; Guyoneaud, R.; Goñi-Urriza, M. The bacterial diversity on steam vents from Paricutín and Sapichu volcanoes. Extremophiles 2019, 23, 249-263. [CrossRef]

45. Yabe, S.; Sakai, Y.; Abe, K.; Yokota, A. Diversity of Ktedonobacteria with Actinomycetes-like morphology in terrestrial environments. Microbes Environ. 2017, 32, 61-70. [CrossRef]

46. Ghezzi, D.; Sauro, F.; Columbu, A.; Carbone, C.; Hong, P.-Y.; Vergara, F.; De Waele, J.; Cappelletti, M. Transition from unclassified Ktedonobacterales to Actinobacteria during amorphous silica precipitation in a quartzite cave environment. Sci. Rep. 2021, 11, 1-18. [CrossRef]

47. King, C.E.; King, G.M. Description of Thermogemmatispora carboxidivorans sp. nov., a carbon-monoxide-oxidizing member of the class Ktedonobacteria isolated from a geothermally heated biofilm, and analysis of carbon monoxide oxidation by members of the class Ktedonobacteria. Int. J. Syst. Evol. Microbiol. 2014, 64, 1244-1251. [CrossRef] 
48. Arif, S.; Nacke, H.; Hoppert, M. Metagenome-assembled genome sequences of a biofilm derived from Marsberg Copper Mine. Microbiol. Res. Announ. 2021, 10, e01253-20.

49. Wall, K.; Cornell, J.; Bizzoco, R.W.; Kelley, S.T. Biodiversity hot spot on a hot spot: Novel extremophile diversity in Hawaiian fumaroles. Microbiology 2015, 4, 267-281. [CrossRef]

50. Tassi, F.; Venturi, S.; Cabassi, J.; Capecchiacci, F.; Nisi, B.; Vaselli, O. Volatile organic compounds (VOCs) in soil gases from Solfatara crater (Campi Flegrei, southern Italy): Geogenic source(s) vs. biogeochemical processes. Appl. Geochem. 2015, 56, 37-49. [CrossRef]

51. Ji, M.; Greening, C.; Vanwonterghem, I.; Carere, C.R.; Bay, S.K.; Steen, J.A.; Montgomery, K.; Lines, T.; Beardall, J.; Van Dorst, J.; et al. Atmospheric trace gases support primary production in Antarctic desert surface soil. Nat. Cell Biol. 2017, 552, 400-403. [CrossRef] [PubMed]

52. King, G.M.; Weber, C.F.; Nanba, K.; Sato, Y.; Ohta, H. Atmospheric CO and hydrogen uptake and CO oxidizer phylogeny for Miyakejima, Japan volcanic deposits. Microb. Environ. 2008, 23, 229. [CrossRef] [PubMed]

53. Yang, J.; Zhou, E.; Jiang, H.; Li, W.; Wu, G.; Huang, L.; Hedlund, B.P.; Dong, H. Distribution and diversity of aerobic carbon monoxide-oxidizing bacteria in geothermal springs of China, the Philippines, and the United States. Geomicrobiol. J. 2015, 32, 903-913. [CrossRef]

54. Arce-Rodríguez, A.; Puente-Sánchez, F.; Avendaño, R.; Martínez-Cruz, M.; De Moor, J.M.; Pieper, D.H.; Chavarría, M. Thermoplasmatales and sulfur-oxidizing bacteria dominate the microbial community at the surface water of a $\mathrm{CO}_{2}$-rich hydrothermal spring located in Tenorio Volcano National Park, Costa Rica. Extremophiles 2019, 23, 177-187. [CrossRef] [PubMed]

55. De Miera, L.E.S.; Arroyo, P.; de Luis Calabuig, E.; Ansola, G. Effects of varying $\mathrm{CO}_{2}$ flows on bacterial communities in mesocosms created from two soils. Int. J. Greenh. Gas. Control. 2016, 46, 205-214. [CrossRef]

56. McIlroy, S.J.; Kirkegaard, R.H.; Dueholm, M.S.; Fernando, E.; Karst, S.M.; Albertsen, M.; Nielsen, P.H. Culture-independent analyses reveal novel Anaerolineaceae as abundant primary fermenters in anaerobic digesters treating waste activated sludge. Front. Microbiol. 2017, 8, 1134. [CrossRef] [PubMed]

57. Kochetkova, T.V.; Toshchakov, S.V.; Zayulina, K.S.; Elcheninov, A.G.; Zavarzina, D.G.; Lavrushin, V.Y.; Bonch-Osmolovskaya, E.A.; Kublanov, I.V. Hot in Cold: Microbial life in the hottest springs in permafrost. Microorganisms 2020, 8, 1308. [CrossRef]

58. Nishihara, A.; Matsuura, K.; Tank, M.; McGlynn, S.E.; Thiel, V.; Haruta, S. Nitrogenase activity in thermophilic chemolithoautotrophic bacteria in the phylum Aquificae isolated under nitrogen-fixing conditions from Nakabusa Hot Springs. Microb. Environ. 2018, 33, 394-401. [CrossRef]

59. Rainey, F.A.; Nobre, M.F.; Schumann, P.; Stackebrandt, E.; Da Costa, M.S. Phylogenetic diversity of the Deinococci as determined by 16 S ribosomal DNA sequence comparison. Int. J. System. Evol. Microbiol. 1997, 47, 510-514. [CrossRef] [PubMed]

60. Gerber, E.; Bernard, R.; Castang, S.; Chabot, N.; Coze, F.; Dreux-Zigha, A.; Hauser, E.; Hivin, P.; Joseph, P.; Lazarelli, C.; et al. Deinococcus as new chassis for industrial biotechnology: Biology, physiology and tools. J. Appl. Microbiol. 2015, 119, 1-10. [CrossRef] [PubMed]

61. Sumner, D.; Hawes, I.; Mackey, T.J.; Jungblut, A.D.; Doran, P.T. Antarctic microbial mats: A modern analog for Archean lacustrine oxygen oases. Geology 2015, 43, 887-890. [CrossRef]

62. Hamilton, T.L.; Klatt, J.M.; de Beer, D.; Macalady, J.L. Cyanobacterial photosynthesis under sulfidic conditions: Insights from the isolate Leptolyngbya sp. strain hensonii. ISME J. 2018, 12, 568-584. [CrossRef]

63. De Luca, P.; Taddei, R.; Varano, L. Cyanidioschyzom merolae: A new alga of thermal acidic enviroments. J. Plant Taxon. Geogr. $1978,33$.

64. Merola, A.; Castaldo, R.; De Luca, P.; Gambardella, R.; Musacchio, A.; Taddei, R. Revision of Cyanidium caldarium. Three species of acidophilic algae. G. Bot. Ital. 1981, 115, 189-195. [CrossRef]

65. Yoon, H.S.; Ciniglia, C.; Wu, M.; Comeron, J.M.; Pinto, G.; Pollio, A.; Bhattacharya, D. Establishment of endolithic populations of extremophilic Cyanidiales (Rhodophyta). BMC Evol. Biol. 2006, 6, 78. [CrossRef]

66. Kühn, J.; Ruess, L. Effects of resource quality on the fitness of collembola fed single and mixed diets from the green and brown food chain. Soil Biol. Biochem. 2021, 154, 108156. [CrossRef]

67. Scheu, S.; Folger, M. Single and mixed diets in Collembola: Effects on reproduction and stable isotope fractionation. Funct. Ecol. 2004, 18, 94-102. [CrossRef]

68. Haubert, D.; Häggblom, M.M.; Langel, R.; Scheu, S.; Ruess, L. Trophic shift of stable isotopes and fatty acids in Collembola on bacterial diets. Soil Biol. Biochem. 2006, 38, 2004-2007. [CrossRef]

69. Mehlhorn, H. Encyclopedia of Parasitology, 4th ed.; Springer: Berlin/Heidelberg, Germany, 2016. 